\title{
Perceived pedestrian environment functional factors which are associated with walking in Tropical University Campus
}

\begin{abstract}
During the last decade transportation in Malaysia has been changed and stepped forward into motorized-transportation. However, Malaysian government started to move toward sustainable society, which sustainable transportation is one of the parts. University Putra Malaysia's (UPM) Serdang campus is mainly dependent on the personal cars and shuttle buses. Furthermore, routes in the campus mainly planned for the vehicles rather than pedestrians and cyclists. This study was trying to examinefunctional factors that encourage UPM's students to do pedestrian activities in the campus.In this study quantitative method through questionnaire survey has selected to assess the variables on a sample of subject. The result showed that students were more looking for accessibility and connectivity, and they were more expected to have particularly pedestrian shortcuts. This research can be helpful for the UPM's policy makers and master planners to facilitate in documenting pedestrian environment literature, as well as help urban designers and city planners to have a deeper perspective of pedestrian perceptions and opinions in tropical climate and particularly Malaysia.
\end{abstract}

Keyword: Functional factors; Pedestrian environment; University Campus; Walking 